\title{
CONSTRUÇÃO DE UM ÍNDICE DE QUALIDADE DO EMPREGO NAS MICRORREGIÕES DO RIO GRANDE DO SUL, 1990-2010
}

\section{CONSTRUCTION OF AN INDEX OF QUALITY OF THE JOB IN THE MICROREGIONS OF THE RIO GRANDE DO SUL, $1990-2010$}

Daisy Catiane Schardosim Reck

Pontifícia Universidade Católica do Rio Grande do Sul - Porto Alegre - RS Brasil

\section{Adelar Fochezatto}

Pontifícia Universidade Católica do Rio Grande do Sul - Porto Alegre - RS Brasil

\begin{abstract}
Resumo: Este trabalho tem por objetivo verificar a qualidade do emprego nas microrregiões do Estado do Rio Grande do Sul no período de 1990 a 2010 . Para tanto, foram selecionadas três variáveis: a remuneração média dos trabalhadores, o grau de instrução e o tempo médio de emprego. Usando o Valor Índice Médio - VIM como método de classificação, constatou-se que a microrregião de Caxias do Sul apresentou a melhor qualidade do emprego no período em questão, situando-se, em todos os anos na primeira categoria.
\end{abstract}

Palavras-chave: Economia do trabalho. Qualidade do emprego. Economia regional.

Abstract: This work aims to verify the quality of employment in the regions of Rio Grande do Sul from 1990 to 2010. To this end, we selected three variables: the average pay of workers, the educated and the average time of employment. Using Value Index Average (VIM) as a method of classification, it was found that the microCaxias do Sul had the best quality of employment in the period in question, standing every year in the first category.

Keywords: Labor economics. Quality of employment. The regional economy.

\section{INTRODUÇÃO}


As economias em desenvolvimento apresentam, em geral, altos índices de desigualdade. As condições de vida e de rendimentos variam significativamente entre diferentes grupos que compõem a sociedade. No mercado de trabalho essas desigualdades são manifestadas pela grande heterogeneidade das características do trabalho, sendo esta uma especificidade marcante no caso brasileiro. Tal heterogeneidade aumenta sua significância principalmente na presença das categorias de trabalhadores autônomos, empregados domésticos e trabalhadores sem remuneração. (BASTOS, 2010).

O mercado de trabalho do Brasil apresentou, ao longo das últimas décadas, significativas alterações, sendo que parte delas esteve diretamente relacionada às mudanças políticas e econômicas que se intensificaram a partir da década de 1990. Dentre as modificações, convém destacar: a abertura comercial do país; o processo de reestruturação produtiva; a estabilização econômica alcançada com o Plano Real; e a mudança cambial. Com isso, apresentaram-se transformações importantes nas formas de produção e organização do trabalho.

Nesse contexto, sabe-se que as grandes mudanças econômicas afetam diretamente $o$ mercado de trabalho, podendo alterar expressivamente o número de empregos. Neste estudo há uma breve descrição das principais alterações do mercado de trabalho brasileiro e do Rio Grande do Sul, sendo que seu objetivo central é elaborar um indicador de qualidade do emprego nas microrregiões do Rio Grande do Sul. Essa qualidade resulta da aglutinação de três variáveis: remuneração média do trabalho, número médio de anos de estudo e tempo médio de emprego dos trabalhadores em cada região.

Para tanto, além desta introdução, o trabalho apresenta mais quatro seções. Na seção dois, é feita uma revisão bibliográfica sobre as mudanças do mercado de trabalho no Brasil e no Rio Grande do Sul nas últimas décadas. Na terceira seção, é apresentada a metodologia empregada para elaborar o índice de qualidade do emprego nas microrregiões do Rio Grande do Sul. Na seção quatro, são apresentados os resultados encontrados e, na última seção, aparecem as conclusões gerais do trabalho. 


\section{MUDANÇAS SOCIOECONÔMICAS NO RIO GRANDE DO SUL NAS ÚLTIMAS DÉCADAS}

\subsection{Mudanças demográficas e econômicas}

Considerando a divisão geográfica oficial do Instituto Brasileiro de Geografia e Estatística - IBGE, além da divisão municipal, que atualmente possui 497 municípios, o Estado do Rio Grande do Sul está dividido em sete mesorregiões, 35 microrregiões homogêneas e 22.728 setores censitários (5.724 rurais e 17.004 urbanos). Nesta pesquisa, optou-se pela regionalização das microrregiões homogêneas. Descartou-se trabalhar com municípios porque muitos trabalhadores moram em um município e trabalham em outro, o que poderia levar a conclusões distorcidas. A Tabela 1 apresenta as principais informações econômicas e demográficas (população, PIB, PIB per capita e IDESE) do Rio Grande do Sul (última linha) e de cada uma das suas 35 microrregiões homogêneas.

Segundo os dados da FEE, o Rio Grande do Sul, em 2010, apresentava uma população total de 10.693 .929 habitantes, representando $5,61 \%$ da população total do país. Essa participação, segundo Bandeira (2010), tem mostrado uma tendência de queda ao longo do tempo ${ }^{1}$. A partir da década de 1980, o crescimento médio anual da população do estado tem se apresentado abaixo da média brasileira devido a um declínio relativamente maior dos níveis de fecundidade.

Outro componente demográfico que se alterou nas últimas décadas no estado é o aumento da expectativa de vida ao nascer. Estas alterações apresentaram seus efeitos na estrutura etária da população, uma vez que entre os anos de 1970 a 2000 houve uma diminuição do peso da população jovem e um aumento da proporção de adultos e idosos (JARDIM, 2010).

No que diz respeito ao Produto Interno Bruto - PIB, o Rio Grande do Sul tem mantido, ao longo das últimas décadas, o quarto maior PIB

\footnotetext{
1 Na década de 1980, a taxa média de crescimento populacional do Rio Grande do Sul era de $1,48 \%$ ao ano, enquanto a média brasileira era de 1,93\%. Nos anos 90, esse acontecimento repetiu-se, sendo de $1,23 \%$ para o Estado e de 1,63 para o país (BANDEIRA, 2010).
} 
do país. No ano de 2008, a participação do PIB gaúcho no total do Brasil foi de $6,58 \%$. Em relação à área territorial, são $281.748,5 \mathrm{~km}^{2}$, ou seja, $3,31 \%$ do território nacional.

Tabela 1. Informações econômicas e demográficas das microrregiões do Rio Grande do Sul em anos selecionados

\begin{tabular}{|c|c|c|c|c|}
\hline & & População & & \\
\hline & PIB (R\$ mil) & Total & PIB per capita (R\$) & IDESE \\
\hline Microrregiões & 2008 & 2010 & 2008 & 2008 \\
\hline Cachoeira do Sul & 1.911 .674 & 152.969 & 12.100 & 0,713 \\
\hline Camaquã & 1.636 .699 & 130.448 & 12.502 & 0,741 \\
\hline Campanha Central & 2.130 .814 & 184.948 & 11.323 & 0,754 \\
\hline Campanha Meridional & 2.029 .588 & 173.808 & 11.681 & 0,767 \\
\hline Campanha Ocidental & 6.051 .701 & 364.249 & 16.420 & 0,758 \\
\hline Carazinho & 2.592 .953 & 159.953 & 15.835 & 0,744 \\
\hline Caxias do Sul & 18.452 .787 & 769.617 & 24.858 & 0,821 \\
\hline Cerro Largo & 1.077 .435 & 66.065 & 15.870 & 0,766 \\
\hline Cruz Alta & 3.375 .964 & 150.223 & 21.808 & 0,772 \\
\hline Erechim & 3.695 .705 & 211.653 & 17.014 & 0,751 \\
\hline Frederico Westphalen & 2.143 .978 & 174.605 & 11.759 & 0,686 \\
\hline Gramado-Canela & 4.038 .086 & 296.581 & 13.431 & 0,737 \\
\hline Guaporé & 2.633 .131 & 127.249 & 20.219 & 0,742 \\
\hline ljuí & 3.251 .728 & 183.920 & 17.423 & 0,767 \\
\hline Jaguarão & 646.320 & 53.154 & 11.894 & 0,729 \\
\hline Lajeado-Estrela & 5.449 .844 & 305.560 & 17.766 & 0,741 \\
\hline Litoral Lagunar & 7.044 .127 & 259.638 & 27.222 & 0,789 \\
\hline Montenegro & 3.299 .562 & 202.517 & 16.563 & 0,720 \\
\hline Não-Me-Toque & 1.006 .317 & 42.221 & 23.488 & 0,747 \\
\hline Osório & 3.570 .616 & 341.119 & 10.542 & 0,712 \\
\hline Passo Fundo & 6.472 .097 & 327.703 & 19.634 & 0,761 \\
\hline Pelotas & 5.079 .916 & 482.915 & 10.112 & 0,737 \\
\hline Porto Alegre & 78.496 .493 & 3.628 .029 & 21.168 & 0,802 \\
\hline Restinga Seca & 854.268 & 63.240 & 13.181 & 0,683 \\
\hline Sananduva & 932.319 & 60.636 & 14.926 & 0,724 \\
\hline Santa Cruz do Sul & 6.409 .745 & 320.312 & 19.646 & 0,720 \\
\hline Santa Maria & 4.418 .426 & 363.016 & 11.874 & 0,773 \\
\hline Santa Rosa & 2.576 .975 & 157.276 & 16.389 & 0,771 \\
\hline Santiago & 1.850 .899 & 110.682 & 16.035 & 0,766 \\
\hline Santo Ângelo & 2.974 .411 & 196.971 & 14.740 & 0,759 \\
\hline São Jerônimo & 6.965 .148 & 143.608 & 48.416 & 0,740 \\
\hline Serras de Sudeste & 1.281 .223 & 116.423 & 10.759 & 0,691 \\
\hline Soledade & 729.876 & 71.490 & 9.750 & 0,689 \\
\hline Três Passos & 2.029 .153 & 143.794 & 13.700 & 0,704 \\
\hline
\end{tabular}


Vacaria

2.384 .267

157.337

14.617 0,762

Rio Grande do Sul

199.494.246

10.693.929

18.655

0,772

Fonte: elaboração dos autores. Dados brutos: FEEDADOS/FEE.

O Índice de Desenvolvimento Socioeconômico - IDESE engloba um amplo conjunto de indicadores sociais e econômicos, agregados em quatro dimensões: educação, saúde, renda e saneamento básico. $O$ objetivo desse índice é fornecer o nível de desenvolvimento do estado. O IDESE é resultado da associação, com a mesma ponderação, de quatro blocos de indicadores: domicílio e saneamento, educação, saúde e renda. O IDESE varia de zero a um, gerando três classificações de desenvolvimento: baixo (até 0,499 ), médio (entre 0,500 e 0,799 ) e alto (maiores ou iguais a 0,800) (FEE, 2011).

De acordo com essa classificação, apenas as microrregiões de Caxias do Sul e Porto Alegre apresentam alto nível de desenvolvimento (ver Tabela 1). O objetivo desta apresentação é verificar, posteriormente, a compatibilidade das microrregiões que apresentam alta qualidade do emprego do estado, com as microrregiões de maior índice de desenvolvimento.

\subsection{Reestruturação produtiva e o mercado de trabalho no Brasil}

Sabe-se que a economia brasileira apresentou significativas transformações nas últimas três décadas, ocasionando expressivas alterações no mercado de trabalho. Primeiramente, na década de 1980, o país atravessou uma crise econômica que caracterizou este período como "a década perdida", dado o baixo crescimento econômico apresentado, a crise da dívida externa e a elevada inflação. Dentre as modificações com maior relevância dessa fase, destaca-se o esgotamento do modelo de desenvolvimento vigente na economia brasileira desde a década de 1930: o modelo de substituição de importações².

Durante as décadas de atuação do Estado Desenvolvimentista, foram vivenciadas elevadas taxas de crescimento do produto,

\footnotetext{
2 A saber, durante as décadas de 1930 a 1970, o Estado brasileiro atuou fortemente na economia, assumindo o papel de financiador, empreendedor, coordenar e regulamentador. O principal objetivo da intensa participação do Estado era promover a industrialização do país, o que o caracterizou como o Estado Desenvolvimentista.
}

Redes (St. Cruz Sul, Online), v. 20, n 3 - Suplemento, p. $226-240$, set./dez. $201 \not 30$ 
ocasionando uma criação relativamente grande de novos empregos. No entanto, as dificuldades apresentadas na crise da década de 1980 introduziram mudanças, principalmente no que se refere ao papel do Estado na economia. Tais modificações foram consubstanciadas na Reforma do Estado da década posterior, que apresentou a necessidade de redefinição do papel do Estado na economia.

Segundo Fligenspan (2000), a palavra que resume a economia brasileira da década de 1990 é "reestruturação", estando relacionada a uma série de fatores, tais como: o processo de abertura comercial, o programa de privatizações, a desnacionalização do capital produtivo e a estabilização monetária. Sendo assim, "é impossível falar desses anos sem considerar a reestruturação do sistema produtivo, do mercado de trabalho, das finanças públicas, dentre tantas outras mudanças significativas observadas nesses poucos anos" (FLIGENSPAN, 2000, p. 5).

Dessa maneira, a evolução do mercado de trabalho brasileiro esteve condicionada às grandes alterações do cenário econômico. Devese salientar que tais mudanças estavam inseridas em um amplo programa de ajuste - de reestruturação - que, em geral, tem implicações sociais. De acordo com Fligenspan (2000, p. 10), "a face mais dramática desses ajustes se deu sobre o mercado de trabalho, onde se verificaram inequívocos sinais de deterioração". Isso porque o conjunto de mudanças ocorridas na década de 1990 culminou em um processo de reestruturação do parque produtivo do país. As empresas passaram a inovar e promoveram mudanças organizacionais e terceirização das atividades. O reflexo dessas alterações no mercado de trabalho foi: aumento significativo do desemprego, aumento da ocupação por conta própria e aumento dos trabalhadores assalariados sem carteira assinada. Assim, foi nesse período que se acentuou um processo conhecido nos estudos econômicos como precarização do trabalho (BASTOS, 2010).

Contudo, nos anos 2000 há uma retomada do movimento de estruturação do mercado de trabalho do país. Especificamente, a partir de 2004, quando o país passa a apresentar melhores taxas de crescimento do PIB, ocorre um aumento da capacidade de geração de empregos, em especial do emprego com carteira assinada, possibilitando, além da redução do desemprego, uma redução da precarização do mercado de trabalho (BASTOS, 2010). 


\subsection{O mercado de trabalho no Rio Grande do Sul}

Além das mudanças demográficas citadas anteriormente, é relevante apresentar a composição da oferta de trabalho do Rio Grande do Sul para compreensão do comportamento do mercado de trabalho do estado. De acordo com Bastos (2010), é a interação entre a População em Idade Ativa - PIA e a População Economicamente Ativa - PEA que determinará o comportamento da oferta de trabalho da região ${ }^{3}$.

Convém salientar que diversos fatores, sejam eles econômicos ou sociais, podem influenciar na iniciativa ou na necessidade das pessoas participarem da atividade econômica. Isso significa que um aumento da taxa de crescimento da população ou, mais especificamente, um acréscimo da taxa de crescimento da PIA não refletirá, necessariamente, em uma variação simétrica da PEA (XAVIER SOBRINHO, 2000).

Em relação à PIA, no período de 1992-1999, o Rio Grande do Sul apresentou uma taxa média anual de crescimento de $1,3 \%$, enquanto que, no período 2001-2007, houve uma aceleração deste ritmo, sendo de $1,8 \%$ o crescimento apresentado. Já a PEA demonstrou um ritmo de crescimento mais modesto no primeiro período de analise (1992-1999), sendo de $0,6 \%$ ao ano quando comparado ao segundo período (20012007), que foi de $1,7 \%$ ao ano (BASTOS, 2010).

Segundo Bastos (2010), uma possível explicação para o baixo ritmo de crescimento da PEA nos anos de 1990 está relacionada à falta de capacidade de geração de oportunidades de trabalho, que foi um fato marcante na década. Com isso, os potenciais trabalhadores - membros da PIA - sentiram menos interesse em participar do mercado de trabalho do estado.

De fato, as décadas de 1990 e 2000 apresentam características bem distintas em relação ao mercado de trabalho. Os anos de 1990 apresentaram mudanças econômicas adversas para geração de oportunidades de emprego. A abertura comercial com valorização cambial gerou o processo de reestruturação produtiva, com impacto negativo sobre o mercado de trabalho. $\mathrm{Na}$ década subsequente, $\mathrm{O}$

\footnotetext{
${ }^{3}$ A PIA corresponde ao conjunto de indivíduos de dez anos ou mais de idade, enquanto a PEA é a parcela da PIA que participa do mercado de trabalho.
} 
contexto econômico tornou-se mais favorável para a retomada da geração de oportunidades de emprego, influenciado pelo processo de desvalorização cambial ocorrido em 1999.

Os dados da taxa de ocupação no estado corroboram tais afirmações. A taxa média anual de crescimento da ocupação no Rio Grande do Sul, no período de 1992-1999, foi de apenas 0,2\%, ou seja, uma capacidade muito pequena de geração de oportunidades de trabalho. Em relação ao período 2001-2007, a taxa média de crescimento foi de $1,7 \%$ ao ano, demonstrando uma melhora significativa na geração de oportunidades. A taxa de desemprego do estado acompanhou os fatores adversos da década de 1990. O crescimento da PEA, aliado à baixa capacidade de geração de empregos no estado, fez com que a taxa de desemprego fosse de $4,3 \%$, em 1992 , para 7,3\%, em 1999 (BASTOS, 2010).

Portanto, o mercado de trabalho gaúcho apresentou fortes sinais de deterioração na década de 1990. O baixo crescimento ocupacional conduziu a economia praticamente a uma situação de estagnação da capacidade de geração de empregos. A elevação do desemprego,, associada ao contexto econômico adverso do período, gerou um fenômeno conhecido como precarização do trabalho. Em contrapartida, na década de 2000, houve uma retomada da criação de oportunidades de trabalho, gerando efeitos positivos no mercado de trabalho.

\section{METODOLOGIA}

Conforme exposto, a área de estudo deste trabalho é o Estado do Rio Grande do Sul e a regionalização escolhida para análise são as microrregiões do estado. A pesquisa foi aplicada para os anos de 1990, 1995, 2000, 2005 e 2010. A base de dados foi extraída das informações disponibilizadas pelo Ministério do Trabalho e Emprego - MTE: estatísticas da RAIS 4 e do CAGED 5 .

Desse banco de dados foram selecionadas três variáveis: (a) faixa de remuneração média; (b) grau de instrução; (c) faixa de tempo de emprego do vínculo informado. Sabe-se que o rendimento e o tempo de

\footnotetext{
${ }^{4}$ Relação Anual de Informações Sociais.

5 Cadastro Geral de Empregados e Desempregados.
} 
estudo influenciam positivamente a qualidade do emprego. Já a rotatividade, que diminui o tempo de emprego, apresenta uma relação inversa. Tendo em vista que as variáveis são disponibilizadas em faixas, tornou-se necessário, primeiramente, obter a média ponderada para cada uma das variáveis.

Segundo Barros e Mendonça (1995), as relações de trabalho são complexas na medida em que envolvem aspectos que não são completamente observáveis. Por isso, a qualidade entre os postos de trabalho difere em relação a um grande número de dimensões. No entanto, sob o ponto de vista do trabalhador, algumas dessas diferenças podem ser irrelevantes. Frente a essa situação, os estudos sobre o tema tendem a seguir dois caminhos: levantar informações primárias por meio de aplicação de questionários aos trabalhadores ou utilizar variáveis supostamente relevantes como indicativas de qualidade do emprego. Esse segundo caminho é o que será seguido neste estudo.

Muitas são as variáveis que poderiam ser utilizadas para a construção do indicador. Entre elas, são amplamente usadas, a remuneração, o grau de instrução e o tempo de emprego ou rotatividade. Além dessas, aparecem com frequência o grau de formalização dos trabalhadores e participação de empregados na indústria. A formalização não é utilizada neste trabalho, pois o indicador construído baseia-se apenas nos trabalhadores formais. A participação de empregados na indústria também não foi utilizada, pois ele representa, em si, um indicador de qualidade, já que os trabalhadores desse segmento, em geral, apresentam melhor remuneração, maior grau de instrução e menor rotatividade. Assim, as características dos empregos da indústria são capturadas pelas outras variáveis utilizadas.

Vários trabalhos utilizam essas variáveis como indicadoras de qualidade do emprego. Entre os aplicados para o Brasil, destacam-se Gonzaga (1998) e Sabóia (2000). Gonzaga (1998) analisou especificamente o problema da rotatividade e sua importância para explicar a baixa qualidade do emprego no Brasil. Seu argumento principal é o de que a qualidade depende fundamentalmente da produtividade que, por sua vez, depende da educação em geral e de treinamento dentro do ambiente de trabalho. Uma alta rotatividade tende a diminuir o treinamento, prejudicando a qualidade do emprego. 
Sabóia (2000) desenvolve um índice sintético para o mercado de trabalho urbano no Brasil, inspirado na metodologia do Índice de Desenvolvimento Humano - IDH. Ele incorpora nove estatísticas, abrangendo os mais diversos aspectos do mercado de trabalho e aplica aos dados das seis regiões metropolitanas cobertas pela Pesquisa Mensal de Emprego do IBGE na década de 1990. Seus resultados mostram tendência de deterioração do mercado de trabalho no período. Por outro lado, ele confirma a situação mais desfavorável das regiões metropolitanas situadas no Nordeste comparativamente ao Sul/Sudeste.

O método de classificação escolhido para determinar e ordenar a qualidade do emprego nas microrregiões do estado foi o VIM (SHAW e WHEELER, 1994). Tal metodologia inicia com o processo de padronização dos dados, a qual foi feita usando a expressão:

$$
\mathrm{Z}_{\mathrm{ij}}=\left(\mathrm{X}_{\mathrm{ij}}-\mu_{\mathrm{i}}\right) / \sigma_{\mathrm{i}}
$$

onde: $Z_{\mathrm{ij}}$ é o valor padronizado da variável i na região $\mathrm{j}, X_{\mathrm{ij}}$ é o valor observado da variável i na região $\mathrm{j}, \mu_{\mathrm{i}}$ é o valor médio da variável i em todas as microrregiões e $\sigma_{i}$ é o desvio padrão da variável $i$ em todas as microrregiões. Após a padronização, todas as variáveis passaram a ter média igual a zero e desvio padrão igual a um. Esses valores foram agrupados em seis classes, como pode ser visto na coluna dois do Quadro 2.

Após a classificação das variáveis é possível calcular o Valor Índice Médio - VIM de cada microrregião. Isso é feito mediante a soma dos valores das classes de cada região nas diferentes variáveis que fazem parte do índice e dividindo pelo número de variáveis que compõem este índice. Ou seja:

$$
\mathrm{VIM}_{\mathrm{j}}=\sum_{\mathrm{i}} \mathrm{C}_{\mathrm{ij}} / \mathrm{n}
$$

onde $C_{i j}$ é a classificação da variável i na região j e $n$ é o número de variáveis no índice. Portanto, todas as variáveis têm o mesmo peso na composição do indicador. Com isso, é possível ordenar e categorizar as microrregiões de acordo com o VIM alcançado. Sendo que, quanto mais próximo de seis, maior será a qualidade relativa do emprego na região 
analisada. Esse processo possibilita a classificação dos resultados $^{6}$, conforme exposto no Quadro 2.

\section{Quadro 2. Classificação dos resultados padronizados}

\begin{tabular}{|c|c|c|}
\hline $\begin{array}{c}\text { Intervalos das variáveis } \\
\text { padronizadas } \\
\left(\mathbf{Z}_{\mathrm{ij}}\right)\end{array}$ & $\begin{array}{c}\text { Classificação } \\
\left(\mathbf{C}_{\mathrm{ij}}\right)\end{array}$ & Interpretação \\
\hline Menor que $-1,000$ & 1 & Muito abaixo da média \\
\hline Entre $-1,000$ e $-0,500$ & 2 & Abaixo da média \\
\hline Entre $-0,499$ e 0,000 & 3 & Abaixo próximo da média \\
\hline Entre 0,001 e 0,500 & 4 & Acima próximo da média \\
\hline Entre 0,501 e 1,000 & 5 & Acima da média \\
\hline Maior que 1,000 & 6 & Muito acima da média \\
\hline
\end{tabular}

Fonte: elaboração dos autores.

\section{ANÁLISE DOS RESULTADOS}

Os resultados completos para o indicador de qualidade do emprego (VIM) e seus componentes em todas as microrregiões e períodos analisados estão no Anexo 2. Nas três primeiras colunas de cada ano, apresenta-se a classificação das variáveis que compõem o VIM, conforme procedimento exposto no Quadro 2. Os valores das variáveis variam de 1 a 6 e quanto mais próximo de seis, melhor a situação da variável na região analisada. O VIM, apresentado na quarta coluna, segue a mesma interpretação: varia de 1 a 6 e quanto maior, melhor.

O Quadro 3 fornece uma síntese dos resultados do Anexo 2, mostrando a distribuição das microrregiões em cinco categorias de valores para o indicador de qualidade do emprego. As regiões que fazem parte da categoria muito acima da média, com VIM no intervalo 5

6 Convém ressaltar que essa classificação foi feita para cada uma das três variáveis nas 35 microrregiões do estado. Contudo, considerando a relação inversa da rotatividade com a qualidade do emprego, a ordem de classificação das categorias foi invertida nesse caso específico. Ou seja, com o ordenamento inverso, atribuiu-se à primeira categoria de classificação (valores menores do que 1) o indicador 6; à segunda categoria (valores entre -1 e $-0,5$ ) o indicador 5 , e assim por diante. 
a 6, podem ser consideradas referências da qualidade do emprego no Rio Grande do Sul. As microrregiões que se classificaram nesse intervalo nos cinco anos analisados foram: em 1990, apenas Caxias do Sul; em 1995, Caxias do Sul, Erechim, Porto Alegre e Santa Cruz do Sul; em 2000, Caxias do Sul, Santa Cruz do Sul e Santa Maria; em 2005, Caxias do Sul, Santa Cruz do Sul e ljuí; e em 2010, apenas Caxias do Sul e Santa Maria. A microrregião de Caxias de Sul foi a única que se classificou na melhor categoria da qualidade do emprego nos cinco anos analisados, sendo o seu melhor desempenho no ano de 2005. 
Quadro 3 - Distribuição das microrregiões do RS nas categorias de qualidade do emprego (VIM) em anos selecionados

\begin{tabular}{|c|c|c|c|c|c|c|}
\hline \multirow{2}{*}{$\begin{array}{c}\text { Qualidade } \\
\text { do emprego } \\
\text { (VIM) }\end{array}$} & \multirow[t]{2}{*}{ Categoria } & \multicolumn{5}{|c|}{ Número de microrregiões } \\
\hline & & 1990 & 1995 & 2000 & 2005 & 2010 \\
\hline 5 a 6 & $\begin{array}{l}\text { Muito acima da } \\
\text { média }\end{array}$ & 1 & 4 & 3 & 3 & 2 \\
\hline 4 a 4,99 & Acima da média & 9 & 7 & 9 & 7 & 10 \\
\hline 3 a 3,99 & Próximo da média & 18 & 15 & 12 & 15 & 15 \\
\hline 2 a 2,99 & Abaixo da média & 5 & 8 & 10 & 9 & 6 \\
\hline 1 a 1,99 & $\begin{array}{l}\text { Muito abaixo da } \\
\text { média }\end{array}$ & 2 & 1 & 1 & 1 & 2 \\
\hline
\end{tabular}

Fonte: elaboração dos autores, a partir do Anexo 2.

Constata-se, também, que não há uma variação muito forte do comportamento da qualidade do emprego nos anos analisados. $O$ número de microrregiões que se situam abaixo da média da qualidade do emprego (com VIM no intervalo entre 1 e 2,99) não apresentou expressivas alterações, sendo a variação máxima entre os anos de 1990, de 7 microrregiões, e o ano de 2000, de 11 microrregiões.

Em linhas gerais, pode-se dizer que os resultados encontrados para o indicador de qualidade do emprego são coerentes porque as regiões que obtiveram as melhores posições nesse indicador são regiões que aparecem bem posicionadas também em termos de PIB per capita e IDESE. No caso específico de Caxias do Sul, a região ficou em primeiro lugar em qualidade de emprego durante todos os anos analisados $\mathrm{e}$ também no IDESE nos anos comparáveis com o VIM. A Figura 1 mostra as relações entre a qualidade do emprego e o IDESE em anos selecionados. 
Figura 1 - Relações entre a qualidade do emprego (VIM) e o IDESE, 2000 e 2010

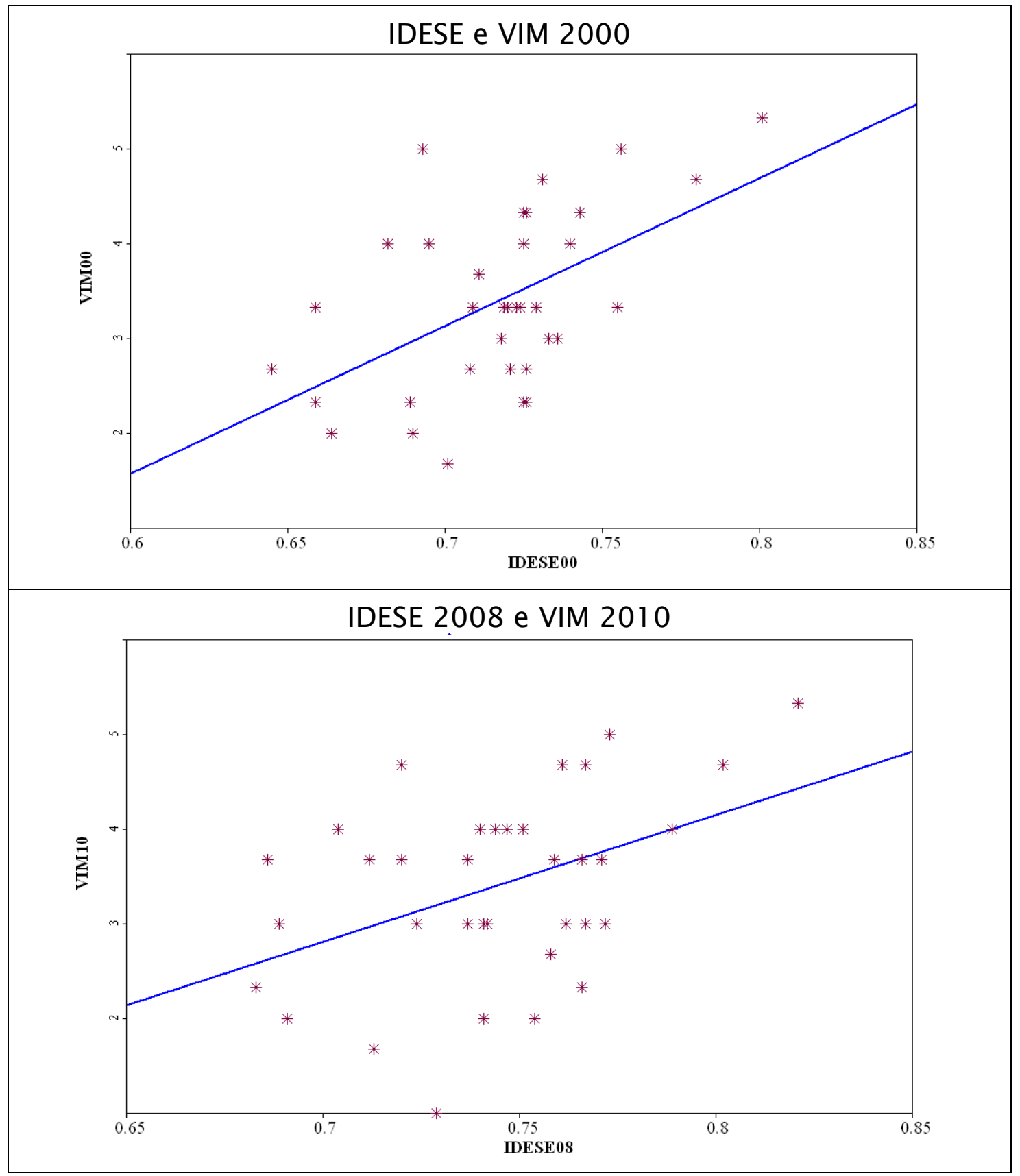

Notas: o VIM de 2010 é comparado com o IDESE de 2008; o coeficiente de correlação do VIM com o PIB per capita de 2008 foi de 0,39 e com o IDESE de 2008 foi de 0,43. 


\section{CONCLUSÕES}

O objetivo deste trabalho foi avaliar a qualidade relativa do emprego nas microrregiões do Rio Grande do Sul. Para isso, foi construído um indicador composto de três dimensões: remuneração média do trabalho, grau de instrução dos trabalhadores e tempo médio de permanência no emprego. A primeira observação a ser feita é que, como era de se esperar, a qualidade do emprego apresentou uma relação consistente com outros indicadores, como o PIB per capita e o IDESE.

As microrregiões que apresentaram a melhor qualidade do emprego foram Caxias do Sul, Porto Alegre, Santa Maria, Santa Cruz do Sul, ljuí e Erechim. Essas regiões, em pelo menos um dos anos estudados, se classificaram na categoria muito superior à média. A microrregião de Caxias do Sul apresentou a melhor qualidade do emprego em todos os anos analisados. É importante destacar que, ao longo da década de 2000, essa microrregião tem sido a primeira colocada também no IDESE.

Os mapas temáticos apresentados no Anexo 1 mostram que a qualidade do emprego nas microrregiões do estado é bastante heterogênea, apresentando um padrão espacial relativamente concentrado. Em linhas gerais, os melhores resultados são encontrados nas microrregiões situadas na metade norte do estado (especialmente no quadrante nordeste). A metade sul concentra as microrregiões classificadas nas categorias muito abaixo da média, sendo destaques negativos as microrregiões de Jaguarão e Serras de Sudeste.

Um aspecto importante que não foi considerado no trabalho é o poder de compra da renda do trabalho. De uma forma geral, pode-se dizer que os maiores salários estão nos maiores centros urbanos e nas regiões onde há concentração da atividade industrial. Nesses locais, o custo de vida também tende a ser maior. No entanto, como o indicador leva em conta outras duas dimensões além da remuneração, problemas como esse acabam ficando diluídos e não comprometem os resultados.

Embora o estudo seja de caráter exploratório, o mapeamento da qualidade do emprego é de muita utilidade, pois traz à tona uma informação sobre uma dimensão do desenvolvimento regional pouco utilizada pelos pesquisadores acadêmicos e pelos formuladores de 
políticas públicas. Apesar da sua importância econômica e social, as informações do mercado de trabalho têm sido sistematicamente desconsideradas na formulação de indicadores de desenvolvimento.

\section{REFERÊNCIAS}

BANDEIRA, M. D. Uma visão demográfica do Estado do Rio Grande do Sul no contexto brasileiro: análise dos principais indicadores demográficos. In: CONCEIÇÃO, O. A. C.; GRANDO, M. Z.; TERUCHKIN, S. U.; FARIA, L. A. E. (Org.). Três Décadas de Economia Gaúcha. Porto Alegre: FEE, 2010.

BARROS, R. P. e MENDONÇA, R. S. P. de. Uma avaliação da qualidade do emprego no Brasil, IPEA: Texto para Discussão n.381, 1995.

BASTOS, R. L. A. Mudanças estruturais no mercado de trabalho do Rio Grande do Sul: 1981-2007. In: CONCEIÇÃO, O. A. C.; GRANDO, M. Z.; TERUCHKIN, S. U.; FARIA, L. A. E. (Org.). Três Décadas de Economia Gaúcha. Porto Alegre: FEE, 2010.

CHAVES, A. L. L.; TONI, M. de; KRELING, N. H.. O mercado de trabalho da RMPA a partir dos anos 90: precarização e (re)estruturação em duas décadas de transformações. In: CONCEIÇÃO, O. A. C.; GRANDO, M. Z.; TERUCHKIN, S. U.; FARIA, L. A. E. (Org.). Três Décadas de Economia Gaúcha. Porto Alegre: FEE, 2010.

FEE. Estatísticas: FEEDADOS. Disponível em:

<http://www.fee.rs.gov.br/feedados>. Acesso em: 6 jul. 2011.

FLIGENSPAN, F. B. (Org.). Economia gaúcha e reestruturação nos anos 90. Porto Alegre: FEE, 2000.

GONZAGA, G. Rotatividade e qualidade do emprego no Brasil. Revista de Economia Política, v.18, n.1, p. 120-140, 1998.

JARDIM, M. de L. T. Tendências demográficas e perspectivas futuras da população gaúcha. In: CONCEIÇÃO, O. A. C.; GRANDO, M. Z.; TERUCHKIN, S. U.; FARIA, L. A. E. (Org.). Três Décadas de Economia Gaúcha. Porto Alegre: FEE, 2010. 
MINISTÉRIO DO TRABALHO E EMPREGO. Base de dados: RAIS e CAGED. Disponível em < http://www.mte.gov.br/pdet/index.asp $>$. Acesso em 20 jun. 2011.

PICHLER, W. A. Mudanças nas relações de trabalho: sindicalismo, greves e negociações coletivas entre 1980 e 2008. In: CONCEIÇÃO, O. A. C.; GRANDO, M. Z.; TERUCHKIN, S. U.; FARIA, L. A. E. (Org.). Três Décadas de Economia Gaúcha. Porto Alegre: FEE, 2010.

SABÓIA, J. Um novo índice para o mercado de trabalho urbano no Brasil. Revista Economia Contemporânea, v.4, n.1, p.123-146, 2000.

SHAW, G. e WHEELER, D. Statistical techniques in geographical analysis. New York: John Wiley \& Sons, $2^{\text {a }}$ Ed. 1994.

TONI, M. de. Precarização do trabalho no Brasil: reversão de tendência no período recente?Textos para Discussão FEE n. 12, 2007.

TONI, M. de. Para onde vai o mercado de trabalho? A tendência à precarização das relações de trabalho - um estudo da Região Metropolitana de Porto Alegre. Porto Alegre: FEE (Tese de doutorado em Sociologia, IFCH-PPGS/UFRGS), 2006, 380p.

XAVIER SOBRINHO, G. G. de F. et al. Mercado de trabalho no Rio Grande do Sul nos anos 90. In: FLIGENSPAN, Flávio Benevett (Org.). Economia gaúcha e reestruturação nos anos 90. Porto Alegre: FEE, 2000.

Submetido em 15/03/2013

Aprovado em 27/11/2015

Sobre os autores

Daisy Catiane Schardosim Reck

Mestre em Economia do Desenvolvimento

Endereço: Rua Maria Bernardina de Oliveira Maciel, 86/401, Vila Eunice Nova. 94920390 - Cachoeirinha - RS - Brasil.

E-mail: catianesr@hotmail.com

Adelar Fochezatto

Redes (St. Cruz Sul, Online), v. 20, n 3 - Suplemento, p. 226 - 240, set./dez. $201 \xi 42$ 
Doutor em Economia

Professor Titular da PUCRS

Endereço: Av. Neusa Brizola, 600/206, Bairro Petrópolis. 90460-230 - Porto Alegre RS - Brasil.

E-mail: adelar@pucrs.br

Anexo 1. Mapas temáticos da qualidade do emprego nas microrregiões do RS, 1990-2010 


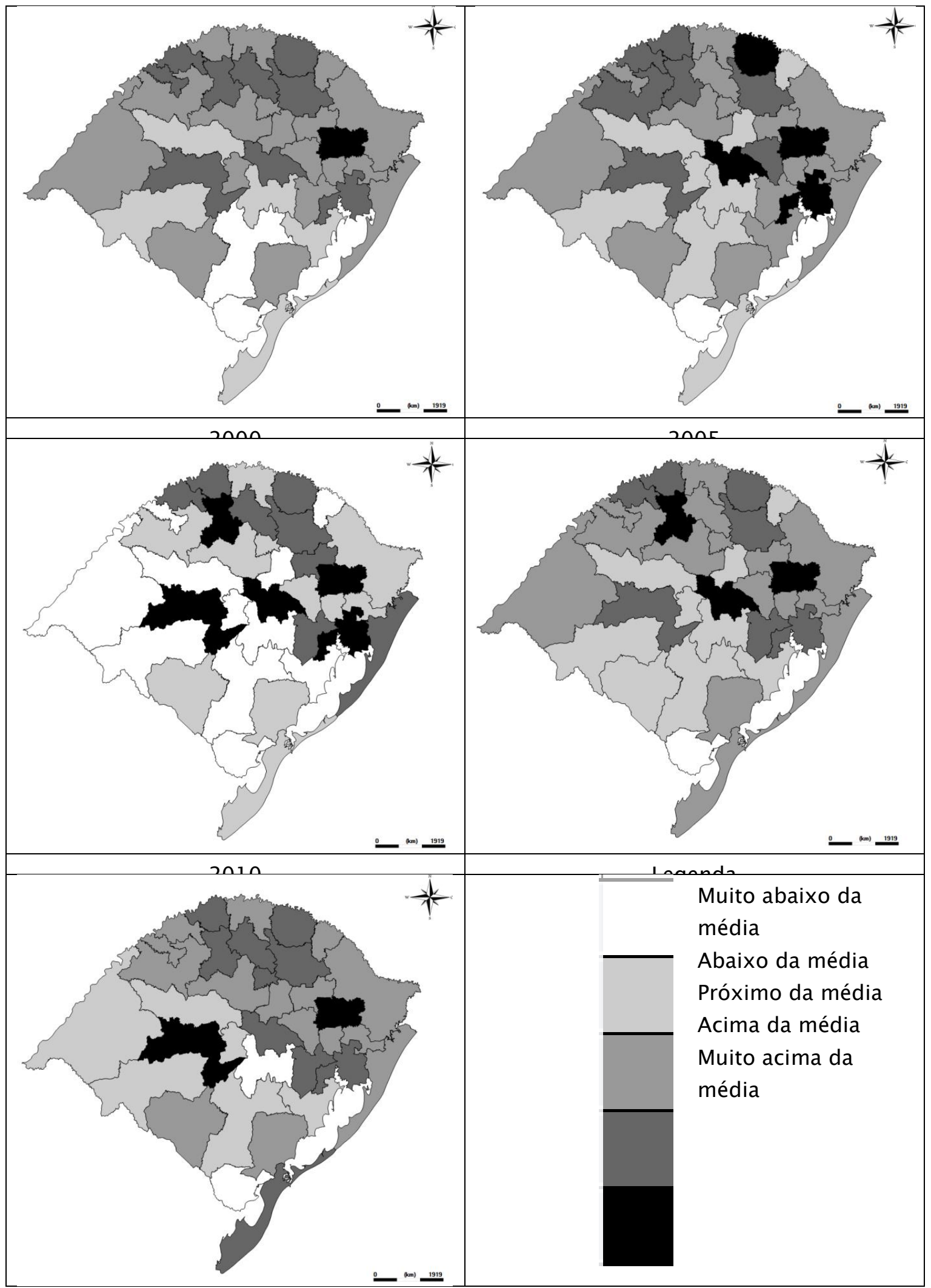

Fonte: Elaboração dos autores. 


\section{Anexo 2. Classificação das microrregiões do RS de acordo com o VIM e seus componentes, anos selecionados}

\begin{tabular}{|c|c|c|c|c|c|c|c|c|c|c|c|c|c|c|c|c|c|c|c|c|}
\hline \multirow[b]{2}{*}{ Micro Regiões do RS } & \multicolumn{4}{|c|}{1990} & \multicolumn{4}{|c|}{1995} & \multicolumn{4}{|c|}{2000} & \multicolumn{4}{|c|}{2005} & \multicolumn{4}{|c|}{2010} \\
\hline & Rem & Temp & Instr & VIM & Rem & Temp & Instr & VIM & Rem & Temp & Instr & VIM & Rem & Temp & Instr & VIM & Rem & Temp & Instr & VIM \\
\hline Caxias do Sul & 6 & 6 & 4 & 5,33 & 6 & 6 & 4 & 5,33 & 6 & 5 & 5 & 5,33 & 6 & 6 & 5 & 5,67 & 6 & 5 & 5 & 5,33 \\
\hline Santa Maria & 6 & 1 & 6 & 4,33 & 4 & 3 & 6 & 4,33 & 6 & 3 & 6 & 5,00 & 6 & 2 & 6 & 4,67 & 6 & 3 & 6 & 5,00 \\
\hline Santa Cruz do Sul & 4 & 4 & 4 & 4,00 & 5 & 5 & 5 & 5,00 & 5 & 5 & 5 & 5,00 & 5 & 6 & 5 & 5,33 & 4 & 5 & 5 & 4,67 \\
\hline ljuí & 3 & 5 & 4 & 4,00 & 4 & 4 & 5 & 4,33 & 5 & 3 & 6 & 4,67 & 5 & 4 & 6 & 5,00 & 4 & 4 & 6 & 4,67 \\
\hline \begin{tabular}{|l} 
Porto Alegre \\
\end{tabular} & 6 & 2 & 6 & 4,67 & 6 & 3 & 6 & 5,00 & 6 & 2 & 6 & 4,67 & 6 & 2 & 6 & 4,67 & 6 & 2 & 6 & 4,67 \\
\hline Passo Fundo & 4 & 4 & 5 & 4,33 & 4 & 4 & 5 & 4,33 & 4 & 4 & 5 & 4,33 & 4 & 5 & 5 & 4,67 & 4 & 5 & 5 & 4,67 \\
\hline São Jerônimo & 6 & 1 & 2 & 3,00 & 6 & 1 & 4 & 3,67 & 6 & 3 & 4 & 4,33 & 6 & 4 & 4 & 4,67 & 6 & 2 & 4 & 4,00 \\
\hline Erechim & 3 & 5 & 4 & 4,00 & 4 & 6 & 5 & 5,00 & 4 & 5 & 4 & 4,33 & 4 & 5 & 4 & 4,33 & 3 & 5 & 4 & 4,00 \\
\hline Três Passos & 4 & 2 & 5 & 3,67 & 4 & 2 & 6 & 4,00 & 4 & 2 & 6 & 4,00 & 3 & 4 & 6 & 4,33 & 3 & 3 & 6 & 4,00 \\
\hline Carazinho & 2 & 5 & 5 & 4,00 & 2 & 4 & 4 & 3,33 & 2 & 4 & 5 & 3,67 & 3 & 4 & 4 & 3,67 & 3 & 5 & 4 & 4,00 \\
\hline Não Me Toque & 4 & 3 & 4 & 3,67 & 4 & 2 & 4 & 3,33 & 4 & 2 & 4 & 3,33 & 4 & 2 & 4 & 3,33 & 4 & 4 & 4 & 4,00 \\
\hline Litoral Lagunar & 6 & 1 & 1 & 2,67 & 6 & 1 & 1 & 2,67 & 6 & 2 & 2 & 3,33 & 6 & 2 & 2 & 3,33 & 6 & 2 & 4 & 4,00 \\
\hline Santa Rosa & 3 & 5 & 5 & 4,33 & 3 & 5 & 5 & 4,33 & 3 & 3 & 6 & 4,00 & 3 & 3 & 6 & 4,00 & 3 & 3 & 5 & 3,67 \\
\hline Osório & 3 & 6 & 2 & 3,67 & 2 & 6 & 2 & 3,33 & 3 & 6 & 3 & 4,00 & 2 & 6 & 3 & 3,67 & 2 & 6 & 3 & 3,67 \\
\hline Frederico Westphalen & 2 & 3 & 6 & 3,67 & 2 & 4 & 5 & 3,67 & 2 & 3 & 5 & 3,33 & 2 & 4 & 5 & 3,67 & 2 & 4 & 5 & 3,67 \\
\hline Pelotas & 4 & 4 & 1 & 3,00 & 4 & 4 & 2 & 3,33 & 4 & 4 & 2 & 3,33 & 4 & 3 & 4 & 3,67 & 4 & 3 & 4 & 3,67 \\
\hline Santo Ângelo & 3 & 3 & 5 & 3,67 & 6 & 1 & 6 & 4,33 & 3 & 2 & 5 & 3,33 & 3 & 2 & 5 & 3,33 & 3 & 3 & 5 & 3,67 \\
\hline Montenegro & 3 & 6 & 1 & 3,33 & 4 & 6 & 1 & 3,67 & 3 & 6 & 1 & 3,33 & 3 & 6 & 1 & 3,33 & 4 & 6 & 1 & 3,67 \\
\hline Cerro Largo & 3 & 4 & 6 & 4,33 & 2 & 2 & 6 & 3,33 & 2 & 1 & 5 & 2,67 & 3 & 1 & 5 & 3,00 & 4 & 2 & 5 & 3,67 \\
\hline Guaporé & 2 & 6 & 3 & 3,67 & 2 & 5 & 3 & 3,33 & 3 & 6 & 3 & 4,00 & 3 & 6 & 2 & 3,67 & 3 & 5 & 1 & 3,00 \\
\hline Lajeado Estrela & 2 & 6 & 2 & 3,33 & 3 & 6 & 3 & 4,00 & 2 & 6 & 2 & 3,33 & 2 & 6 & 2 & 3,33 & 2 & 6 & 1 & 3,00 \\
\hline Cruz Alta & 3 & 3 & 5 & 3,67 & 3 & 3 & 3 & 3,00 & 4 & 2 & 4 & 3,33 & 3 & 3 & 3 & 3,00 & 3 & 3 & 3 & 3,00 \\
\hline Vacaria & 2 & 5 & 2 & 3,00 & 2 & 6 & 2 & 3,33 & 2 & 6 & 1 & 3,00 & 2 & 6 & 1 & 3,00 & 2 & 6 & 1 & 3,00 \\
\hline Gramado Canela & 2 & 6 & 1 & 3,00 & 2 & 6 & 1 & 3,00 & 2 & 6 & 1 & 3,00 & 2 & 6 & 1 & 3,00 & 2 & 6 & 1 & 3,00 \\
\hline Campanha Meridional & 4 & 1 & 5 & 3,33 & 3 & 2 & 4 & 3,00 & 3 & 2 & 4 & 3,00 & 2 & 2 & 4 & 2,67 & 3 & 2 & 4 & 3,00 \\
\hline Soledade & 2 & 4 & 4 & 3,33 & 1 & 3 & 4 & 2,67 & 2 & 3 & 3 & 2,67 & 2 & 3 & 3 & 2,67 & 2 & 3 & 4 & 3,00 \\
\hline Sananduva & 2 & 2 & 5 & 3,00 & 2 & 2 & 4 & 2,67 & 2 & 1 & 3 & 2,00 & 2 & 1 & 4 & 2,33 & 2 & 2 & 5 & 3,00 \\
\hline Campanha Ocidental & 3 & 4 & 4 & 3,67 & 3 & 4 & 3 & 3,33 & 3 & 3 & 2 & 2,67 & 3 & 3 & 3 & 3,00 & 2 & 3 & 3 & 2,67 \\
\hline Restinga Seca & 4 & 1 & 5 & 3,33 & 3 & 2 & 3 & 2,67 & 2 & 2 & 3 & 2,33 & 3 & 1 & 3 & 2,33 & 3 & 1 & 3 & 2,33 \\
\hline Santiago & 2 & 1 & 4 & 2,33 & 2 & 1 & 4 & 2,33 & 2 & 2 & 3 & 2,33 & 2 & 2 & 3 & 2,33 & 2 & 2 & 3 & 2,33 \\
\hline Camaquã & 2 & 5 & 1 & 2,67 & 3 & 5 & 1 & 3,00 & 2 & 5 & 1 & 2,67 & 2 & 4 & 2 & 2,67 & 2 & 3 & 1 & 2,00 \\
\hline
\end{tabular}

Redes (St. Cruz Sul, Online), v. 20, nº 3 - Suplemento, p. 226 - 240, set./dez. 2015245 
Construção de um Índice de Qualidade do Emprego nas Microrregiões do Rio Grande do Sul

\begin{tabular}{|c|c|c|c|c|c|c|c|c|c|c|c|c|c|c|c|c|c|c|c|c|}
\hline Campanha Central & 2 & 3 & 3 & 2,67 & 2 & 3 & 3 & 2,67 & 1 & 3 & 3 & 2,33 & 1 & 3 & 3 & 2,33 & 1 & 3 & 2 & 2,00 \\
\hline Serras de Sudeste & 3 & 1 & 1 & 1,67 & 3 & 2 & 1 & 2,00 & 3 & 2 & 1 & 2,00 & 3 & 2 & 1 & 2,00 & 4 & 1 & 1 & 2,00 \\
\hline Cachoeira do Sul & 2 & 2 & 4 & 2,67 & 3 & 2 & 3 & 2,67 & 3 & 2 & 2 & 2,33 & 3 & 1 & 2 & 2,00 & 2 & 1 & 2 & 1,67 \\
\hline Jaguarao & 2 & 2 & 1 & 1,67 & 2 & 2 & 1 & 1,67 & 2 & 2 & 1 & 1,67 & 1 & 1 & 1 & 1,00 & 1 & 1 & 1 & 1,00 \\
\hline
\end{tabular}

Fonte: elaboração dos autores.

Notas: a) definição das variáveis: Rem=faixa de remuneração média; Temp= tempo de emprego; Instr= grau de instrução; b) as regiões estão ordenadas da maior à menor em qualidade do emprego no ano de 2010; e c) as áreas marcadas em cinza indicam valores do VIM iguais ou maiores que 4 (categorias acima e muito acima da média). 